\title{
Experiment to Internally Measure the Earth's Rotating Speed and the Calculation Comparison with the Relativistic Treatment
}

\author{
Jaroslav Hynecek $^{1}$ \\ ${ }^{1}$ Isetex, Inc., 905 Pampa Drive, Allen, Texas, USA \\ Correspondence: Jaroslav Hynecek, Isetex, Inc., 905 Pampa Drive, Allen, TX 75013, USA. E-mail: \\ jhynecek@netscape.net
}

Received: May 31, 2012 Accepted: July 4, 2012 Online Published: July 20, 2012

doi:10.5539/apr.v4n3p131 URL: http://dx.doi.org/10.5539/apr.v4n3p131

\begin{abstract}
In this paper it is shown that the Earth's rotating speed can be internally measured using the One-way interferometer where the light in one arm of the interferometer travels in an optically dense medium rather than in a vacuum or air. The reason that such a measurement can be made without the null result is the influence of the centripetal force of the Earth's rotation that is present on the Earth's surface as in all rotating platforms and that has to be included into considerations. In all the previous analyses of similar experiments the Earth's rotation was not considered, which leads to various erroneous conclusions. The paper is contrasting the difference between the inclusion of the centripetal force or neglecting it in the analysis of the experiment, which is one of the key points of this paper. The centripetal force compensates for most of the relativistic effects thus allowing the internal motion detection by this type of interferometer. The usual relativistic treatment of this experiment is therefore included as reference for a comparison in this analysis.
\end{abstract}

Keywords: Sagnac effect, the one way speed of light, one-way interferometer, Galilean addition of velocities, internal measurement of Earth's rotation, compensation of relativistic effects by rotation on solid platforms, Michelson-Morley interferometer experiment

\section{Introduction}

There have been many papers written about similar experiments with various arguments that prove or disprove the special relativity theory (SRT) and most of them claim that such a measurement can yield only the null result despite the well known fact that the Sagnac effect, which has a similar underlying physics as this experiment, does not yield the null result. A good review of many such experiments was recently published by Gift (2012) including the correct explanation of why the Michelson-Morley type of experiments typically yield null results. These experiments are the second order type with the interference fringe shift proportional to $v^{2} / c^{2}$, which is very small for the case of the Earth's rotation. The Gift's paper, however, also claims that the aether wind has been detected. This conclusion is not correct as is explained in this paper and as it follows from the recently published paper by Hynecek (2012a). In Hynecek's publication the author has explained that all the previous descriptions and analyses of similar experiments have committed a fundamental error by neglecting the centripetal force effect of the Earth's rotation. When the Earth's rotation is properly considered it is found that the centripetal force and the corresponding inertial centrifugal reaction to it cancel most of the SRT effects and the space-time geometry of the experiment is flat with a simple Galilean addition of velocities. The SRT is still valid, but should not be applied to a system that is not in an inertial motion. The inclusion of the centripetal force allows to detect the Earth's rotation similarly as the ring laser gyroscopes detect the rotation of inertial navigation systems that are based on the Sagnac effect. If the aether wind instead of just the Earth's rotation would be detectable the results would have to also include the Earth's orbital speed, or the Solar system speed in the Milky Way galaxy, or even the Milky Way galaxy recession velocity, which are all much higher than the Earth's rotation speed. These velocities, however, are not detected because these motions are essentially free falls and do not contain centripetal forces generated by the cohesion of solid bodies similarly as in the case of the Earth's surface. The explanation that such motions are not detectable correctly follows from SRT, since these motions are inertial-like following the geodesic lines. An example is the Earth's motion in orbit around the Sun where there is no cohesion centripetal force, except gravitation that would force Earth to orbit and no corresponding centrifugal force that we could sense or measure. This conclusion, however, contradicts the 
findings of Demjanov (2010), who claims the detection of aether velocity of $480 \mathrm{~km} / \mathrm{sec}$. The only possibility of such a high velocity detection is the existence of cohesion forces existing in the Milky Way galaxy arms between its stars. While such forces may exist they have not been detected yet in the star motion, or otherwise theoretically predicted.

In this article the One-Way interferometer will be analyzed including the centripetal and centrifugal forces of the Earth's rotation.

\section{Experimental Setup}

The experimental arrangement of the One-way interferometer is shown in Figure1. The beam splitter $\mathrm{M}_{1}$ divides the incoming laser light beam into the two beam components. The first beam continues to travel straight in the original direction, but in a medium with the index of refraction larger than unity, for example in the quartz rod with the index of refraction $n=1.4585$. The second beam is diverted to travel around this medium and is reflected by the mirrors $\mathrm{M}_{2}$ and $\mathrm{M}_{2}$ to recombine on the second beam splitter $\mathrm{M}_{3}$ to create the interference fringe pattern that is observed on the viewing screen.

In the previously published paper Hynecek (2012b) has shown that due to the presence of the centrifugal force in a rotating platform the Fresnel light dragging coefficient has to be modified and for the light speed in the optically dense medium in the Earth's east-west direction the following formula must be used:

$$
c^{\prime}=c / n+v(1-1 / n)
$$

For the index of refraction $n=1$ it is: $c^{\prime}=c$. When there is no centrifugal force the standard SRT velocity addition formula provides the correct equation for the speed of light as follows:

$$
c^{\prime}=\frac{c / n+v}{1+v / n c} .
$$

Since the time of the photon flight in the optically dense medium is longer than in the air a certain adjustable distance " $\mathrm{a}$ " has been added to the path in the air so that the photons travelling along either path arrive at the mirror $\mathrm{M}_{3}$ at the same time.

The times of the photon flight through the optically dense medium of length $L$ are calculated in the next steps. The time $t_{1}$ corresponds to the case when the centripetal force is considered and the time $t_{1}^{r}$ is for the relativistic treatment when this force is not considered:

$$
t_{1}=\frac{L+v t_{1}}{c / n+v(1-1 / n)}, \quad t_{1}^{r}=\frac{L \sqrt{1-v^{2} / c^{2}}+v t_{1}^{r}}{c / n+v}(1+v / n c) .
$$

Solving these equations for time results in the following:

$$
t_{1}=n L /(c-v) \text { and } t_{1}^{r}=\frac{n L}{c} \frac{1+v / n c}{\sqrt{1-v^{2} / c^{2}}} .
$$

For the parallel portion of the same path in the air the corresponding times of travel are simply:

$$
t_{2}=L /(c-v) \text { and } t_{2}^{r}=\frac{L}{c} \frac{1+v / c}{\sqrt{1-v^{2} / c^{2}}},
$$

where it was substituted for $\mathrm{n}: n=1$. A certain difficulty can present itself when the time of the photon flight has to be calculated in the direction, perpendicular to the light path in the medium. To calculate the light reflection from a tilted and moving mirror is not a simple task. However, this problem can be circumvented by assuming that the distance "a" will be adjusted to a certain value when the flight time difference is set to zero after the interferometer is turned around by $180^{\circ}$ into the direction of $-v$. It is now easy to calculate the time arrival difference when the interferometer is turned back into the direction of $+v$. The time arrival difference: $\Delta t=t_{1}-t_{2}$ of photons that travel along the different paths is:

$$
c \Delta t=\frac{L(n-1)}{1-v / c}-\frac{L(n-1)}{1+v / c}=\frac{2 L(n-1)}{1-v^{2} / c^{2}} \frac{v}{c},
$$

and similarly for the relativistic case without considering the centripetal force effect it is: 


$$
c \Delta t^{r}=2 L\left(\frac{n v / n c}{\sqrt{1-v^{2} / c^{2}}}-\frac{v / c}{\sqrt{1-v^{2} / c^{2}}}\right)=0 .
$$

By considering only the first order terms in the ratio of $v / c$ and normalizing this result to the wavelength of the laser light that is used in the experiment the relative interference fringe shift dependence on the velocity is thus finally calculated to be:

$$
\frac{\Delta \lambda}{\lambda}=\frac{2 L}{\lambda}(n-1) \frac{v}{c}
$$

and for the relativistic case:

$$
\frac{\Delta \lambda^{r}}{\lambda}=0
$$

These are simple formulas with the first order dependency on the ratio of $v / c$ or no dependency at all, which should be relatively easy to observe. This experiment will thus clearly detect the influence of the centripetal force on the outcome and once for all resolve the ongoing dispute about the correctness or incorrectness of the SRT treatment of similar experiments such as the famous Michelson-Morley experiment conducted on the surface of the rotating body such as Earth. The confidence that the formula in Eq.8 provides the correct result when the centripetal force is included can be obtained by comparing it with the well known Sagnac effect formula where the fringe shift is calculated from the relation:

$$
\frac{\Delta \lambda}{\lambda}=\frac{2 L}{\lambda} \frac{v}{c}
$$

When this fringe shift is subtracted from the fringe shift obtained from the Sagnac experiment made in the optically dense medium with the index of refraction $\mathrm{n}$, the same result as derived in Eq. 8 is obtained:

$$
\frac{\Delta \lambda}{\lambda}=\frac{2 L}{\lambda}(n-1) \frac{v}{c} .
$$

The time of flight of RF signals in the direction of the Earth's rotation and against it was experimentally verified by the GPS Ashby (2003). The results agree with the Sagnac effect formula prediction. It is important for the SRT, the Sagnac effect, and the resolution of the Ehrenfest paradox, Hynecek (2012a, 2012b) to be all mutually consistent.

\section{Expected Results}

The interference fringe shift due to the Earth's rotation can now be calculated from Eq.8. It will be assumed that the interferometer is of a desktop size with the arm fabricated from the quartz: $n=1.4585$ having the length of $L=1.0 \mathrm{~m}$. The laser light will be of the green color with the wavelength: $\lambda=532 \mathrm{~nm}$. For the Earth's surface velocity at the equator it is: $v_{e}=465.1 \mathrm{~m} / \mathrm{sec}$. The expected fringe shift is thus equal to:

$$
\frac{\Delta \lambda}{\lambda}=\frac{2 L}{\lambda}(n-1) \frac{v_{e}}{c}=2.674 \text {. }
$$

This amount of the interference fringe shift should be easily observable. For the different experiment latitude than on the equator this result must be, of course, multiplied by the cosine of the latitude.

\section{Conclusions}

In this paper it was clearly shown that it is possible to internally detect the rotation of Earth using the One Way interferometer where the one arm of the interferometer is built in an optically dense medium. The non zero interference fringe shift follows from the fact that the centripetal force of the Earth's rotation cancels most of the relativistic effects as has been confirmed for time by the GPS. In all the previous analyses of similar tests the centripetal force of the Earth's rotation was always neglected. In the Michelson-Morley type of experiments this does not create a problem since this is a second order experiment in $v^{2} / c^{2}$ and the error caused by neglecting the centripetal force is very small. However, in the first order experiments, such as in the one described in this paper, the centripetal force cannot be neglected if the correct results consistent with the Sagnac effect and the SRT are to be obtained. The detection of the nonzero fringe shift in this experiment is thus a clear proof of the influence of the centripetal force of Earth's rotation that should always be included in the analysis. 
The detection of the Earth's rotation by this type of interferometer, however, should not be misinterpreted and confused with the detection of the aether wind.

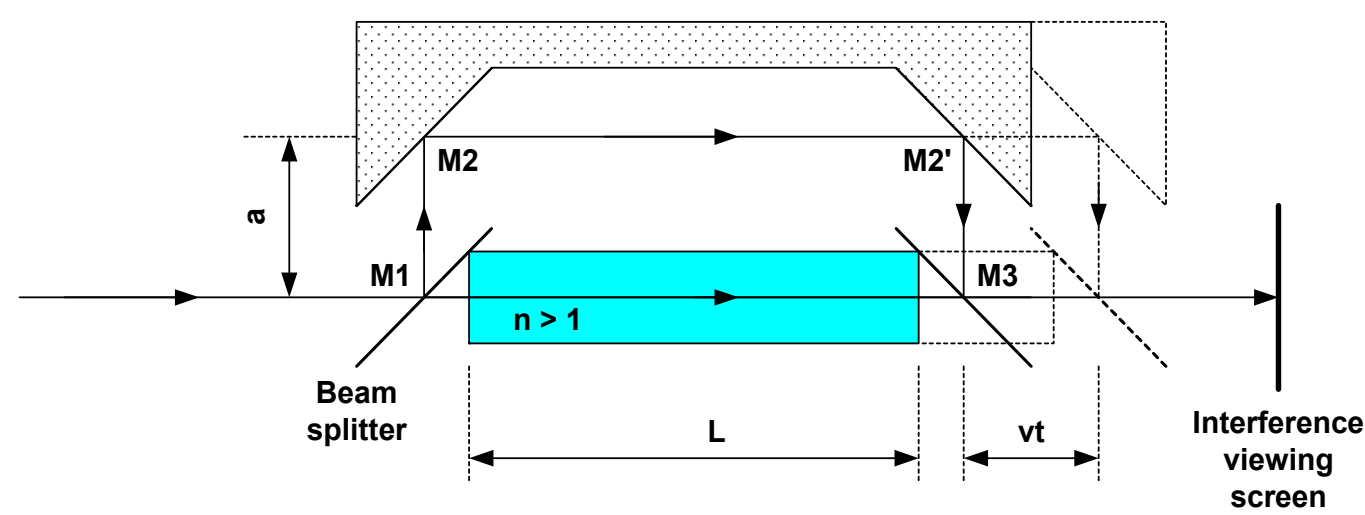

Figure 1. Schematic diagram of the One-Way interferometer experiment

The beam splitter $\mathrm{M}_{1}$ divides the light beam into two beam components. The first beam continues horizontally but in the medium with $n>1$ to the mirror $M_{3}$ and the second beam is reflected around the medium by the mirrors $M_{2}$ and $M_{2}$ to interfere with the first beam at the mirror $M_{3}$. The resulting interference fringes are observed on the viewing screen.

\section{References}

Ashby, N. (2003). Relativity in the Global Positioning System. Living Reviews in Relativity, 6, 1.

Demjanov, V. V. (2010). Michelson Interferometer Operating at Effects of First Order with Respect to v/c (the third method of measuring the speed of "aether wind"). Retrieved 19 April, from arXiv: quant-h/0103103v3

Gift, S. J. G. (2012). Successful Search of Ether Drift in a Modified Michelson-Morley Experiment Using the Global Positioning System (GPS). Applied Physics Research, 4(1), 185. http://dox.doi.org/10.5539/apr.v4n1p185

Hynecek, J. (2012a). Resolution of the Ehrenfest paradox, Sagnac effect, and the Michelson-Morley experiment. Physics Essays, 25(2). http://dx.doi.org/10.4006/0836-1398-25.2.256

Hynecek, J. (2012b). Transversal Fizeau Effect and the GRT. Retrieved from http://www.worldsci.org/pdf/abstracts/abstracts_6511.pdf 